\title{
Increased Prevalence of High Anti-Cladosporium Antibody Titers in Interstitial Lung Diseases
}

\author{
Zenta Watanuki, ${ }^{1}$ Shinji Okada, ${ }^{1}$ Shigeki Chiba, ${ }^{3}$ Katsuhiko Kamei, ${ }^{2}$ \\ Yasuko Suzuki ${ }^{1}$ and Norihiro Yamada ${ }^{1}$ \\ ${ }^{1}$ South Miyagi Medical Center, Ogawara, Miyagi, Japan \\ ${ }^{2}$ Medical Mycology Research Center, Chiba University, Chiba, Japan \\ ${ }^{3}$ Department of Respiratory Medicine, Tohoku University Graduate School of Medicine, Sendai, Japan
}

\begin{abstract}
Interstitial lung diseases (ILDs) represent a large group of different diseases, with a large part comprising idiopathic interstitial pneumonias. Differentiating hypersensitivity pneumonitis (HP), especially its chronic form and other ILDs, is difficult because of similarities in radiological manifestation and clinical course, and the difficulty of identifying causative antigens. We recently experienced a patient with Cladosporiuminduced chronic HP that developed in a household environment, but the cause had been misdiagnosed as idiopathic interstitial pneumonia for several years. This case highlighted the need for measures differentiating HP from idiopathic interstitial pneumonia. In this study, we examined fungal exposure in ILDs using an antibody titer in serum to identify possible fungus-related HP. We measured the antibody titer to Cladosporium spp. in 34 patients with various ILDs, 17 patients with bronchial asthma, and 21 control subjects using an immunofluorescence assay. ILDs included HP (5 patients), idiopathic interstitial pneumonias (21 patients), and ILDs with collagen vascular diseases (8 patients). Results showed a significantly higher tendency for high anti-Cladosporium antibody titers in ILD groups (12 patients out of 34 patients), compared to patients with bronchial asthma $(0 / 17)$ or control subjects $(0 / 21)$. This increase in antibody titers was observed not only in patients with HP, but also in those with idiopathic interstitial pneumonias and those exhibiting collagen vascular diseases with ILDs. This report highlights the pathogenic role of fungal antigens in various ILDs. In conclusion, fungi commonly observed in our living environment such as Cladosporium could be involved in the development of ILDs.
\end{abstract}

Keywords: Cladosporium; extrinsic allergic alveolitis; idiopathic pulmonary fibrosis; indirect fluorescent antibody technique; interstitial lung diseases

Tohoku J. Exp. Med., 2012, 226 (4), 287-291. C 2012 Tohoku University Medical Press

Interstitial lung diseases (ILDs) represent a large group of different diseases, with a large part comprising idiopathic interstitial pneumonias (IIPs). IIPs are further divided phenotypically into several groups such as idiopathic pulmonary fibrosis (IPF), nonspecific interstitial pneumonia (NSIP), and cryptogenic organizing pneumonia (COP). Each of these groups always contains the possibility of including other undiagnosed ILDs such as viral/bacterial pneumonias, drug-induced lung diseases, collagen vascular diseases-related ILDs (CVD-ILDs), and hypersensitivity pneumonitis (HP). Therefore, a revision has been recommended for the diagnosis of ILDs using a more detailed history and more specimens for diagnosis, especially since it is difficult to reach a correct diagnosis in almost all cases with ILDs (American Thoracic Society 2002). Information regarding infectious agents and auto-immune antibodies related to ILDs has been gathered to differentiate viral/bacterial pneumonias and CVD-ILDs from IIPs. However, there have been only limited measures to identify or rule out HP, one of the most difficult diseases to differentiate from IIPs. This may be because of the hundreds of antigens involved in the development of HP, and thus the difficulty of creating a detection system for HP-specific immune responses (Fink et al. 2005).

Previously, we reported a patient with chronic HP caused by Cladosporium species that developed in a household environment, but not in specific environments (Chiba et al. 2009). Although several coincidences helped us to reach a diagnosis of HP in this case after several years of follow up, a large number of patients with HP can be misdiagnosed as IIPs. Furthermore, while studying this patient, all of the control sera drawn from laboratory staff had antibody to Cladosporium spp. up to 640 times according to an immunofluorescence assay. These results suggest the ubiquitous presence of these fungi in our living environments, and the possibility that anyone could develop Cladospo-

Received February 2, 2012; revision accepted for publication March 23, 2012. doi: 10.1620/tjem.226.287

Correspondence: Shinji Okada, M.D., South Miyagi Medical Center, Ogawara Aza-nishi 38-1, Shibata-gun, Miyagi 989-1253, Japan.

e-mail: s.okada@southmiyagi-mc.jp 
rium-related HP at least in this area.

In this study, we measured the antibody titer to Cladosporium spp. in patients with various ILDs to study our hypothesis that a considerable number of patients with undiagnosed Cladosporium-related HP ought to be categorized within the group comprising IIPs. Although the increase in antibody titer is not sufficient for a diagnosis of Cladosporium-related HP because the precipitating antibody to an environmental antigen is sometimes negative in patients with HP and positive in people with antigen exposure without HP, we hope that the results of this study would lead to the better differentiation of HP from IIPs.

\section{Materials and Methods}

Sera from patients with ILDs $(n=34)$ or bronchial asthma (BA) $(n=17)$ who visited or were admitted to our hospital from July 2006 to November 2007 and provided informed consent were kept at $-80^{\circ} \mathrm{C}$ until measurement of the antibody titer. All blood samples were initially drawn from patients for clinical purposes, and the remaining sera were used for this study. The study protocol was approved by the Hospital Ethics Committee of the South Miyagi Medical Center.

All ILD patients without obvious evidence of infection, environmental exposure, or complicated CVD were placed in the IIP group. Among the IIP patients, patients with histological usual interstitial pneumonia (UIP) following surgical biopsy or those with characteristic CT findings and a slowly progressive disease were further classified into IPF. COP was diagnosed by transbronchial lung biopsy. In this study, patients with characteristic CT findings, increased lymphocyte percentage in BALFs, and responsiveness to steroid therapy were classified into the NSIP group. Patients with ILDs showing an increased lymphocyte count in BALFs, evidence of environmental exposure, and an increased lymphocyte response to environmental fungi were diagnosed as HP. All CVD-ILD patients enrolled in this study were patients already diagnosed as CVD by rheumatologists and were referred to us in order to follow up their pulmonary diseases. All met the criteria of the research division of the Health, Labour and Welfare Ministry of Japan. Sera from laboratory staffs were used as a control $(n=21)$.

The antibody titer to Cladosporium cladosporioides in all sera was measured by an immunofluorescence assay (IFA). Antibody titer to Cladosporium herbarum or Leptosphaerulina spp. was measured in sera from ILD patients. Fungi used in this study were isolated from the environments of patients with HP as causative antigens, and identified at the Medical Mycology Research Center, Chiba University (Fig. 1). Fungi were suspended in saline and $50 \mu \mathrm{L}$ of suspension was mounted onto a glass slide, dried overnight in a box, and then fixed. Four-fold dilutions of sera from 10 to 327,680 times were mounted onto a glass slide and incubated for one hour at room temperature. After washing twice with PBS, FITC-labeled rabbit antihuman Kappa Lambda IgA/IgG/IgM (Dako) diluted in PBS was mounted onto a glass slide and incubated for one hour at room temperature. After washing with PBS, the antibody titer was evaluated with a fluorescence microscope (BX51, Olympus, Tokyo, Japan), and the highest dilution with a positive stain was determined to be the antibody titer.

Statistical analysis was performed using Excel Toukei 2002 (Social Survey Research Information Co. Ltd. Tokyo, Japan) run on an Excel spreadsheet. Fisher's exact test was used to compare the prevalence of high antibody titer between each group. Spearman's rank correlation coefficient was used to test the correlation of antibody titer between different fungi. The Student's t-test was used for other comparisons. A $P$-value $<0.05$ was considered statistically significant.

\section{Results}

Antibody titers to Cladosporium cladosporioides in controls ranged from 40 to 640 (median: 160). Thus, we defined 1,280 or higher as a high antibody titer. AntiCladosporium cladosporioides antibody titer was measured in 34 sera from patients with various ILDs, in 17 sera from patients with bronchial asthma (BA), and in 20 healthy controls. ILDs included 5 patients with HP, 8 with CVD-ILD, and 21 with IIPs. IIPs included 16 patients with IPF, 4 with

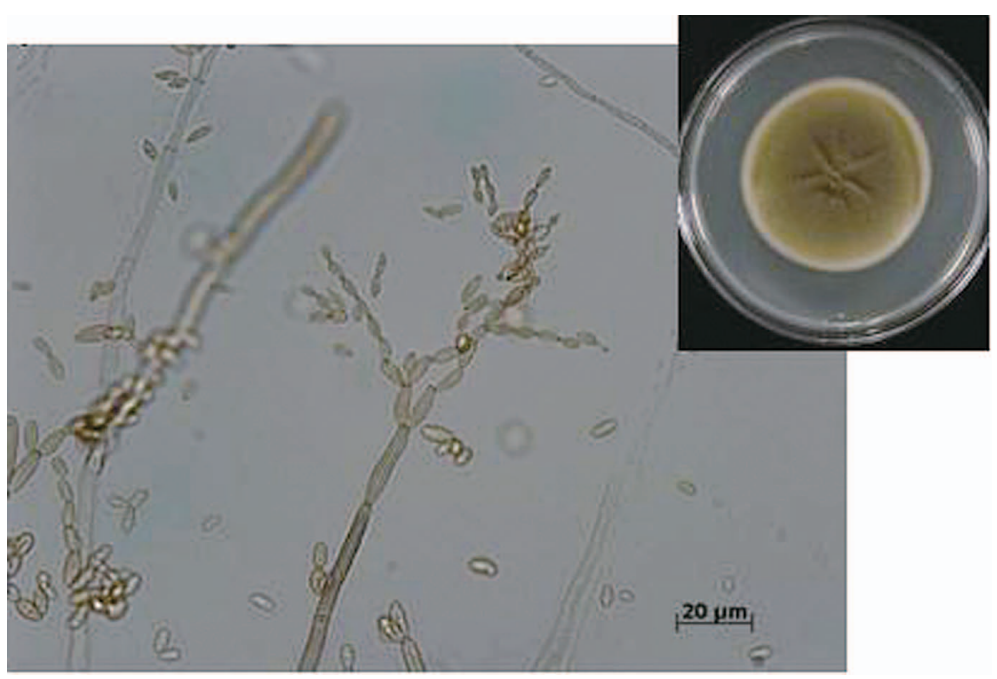

Fig. 1. Cladosporium cladosporioides. Cladosporium is a common fungus frequently encountered in most areas on the earth. The colony of the fungus appears gray to black or very dark green. The fungus used for the antibody assay was isolated from an air conditioner in the office of a patient with HP as a causative antigen. 
Table 1. High anti-Cladosporium cladosporioides antibody titer $(>640)$ in sera of patients with ILDs.

\begin{tabular}{lccc}
\hline & Age & Sex (M:F) & $\begin{array}{c}\text { High Ab Titer/Total } \\
\text { (number of subjects) }\end{array}$ \\
\hline ILDs & $71.6 \pm 13.3$ & $20: 14$ & $12 / 34^{*}$ \\
BA & $58.1 \pm 18.4$ & $6: 11$ & $0 / 17$ \\
control & $37.1 \pm 8.2$ & $21: 0$ & $0 / 21$ \\
\hline
\end{tabular}

$*: p<0.05$

Table 2. High anti-Cladosporium cladosporioides antibody titer (> 640) in sera of ILD patients.

\begin{tabular}{lccc}
\hline & Age & Sex (M:F) & $\begin{array}{c}\text { High Ab } \\
\text { Titer/Total } \\
\text { (number of patients) }\end{array}$ \\
\hline HP & $60.4 \pm 12.9$ & $2: 3$ & $2 / 5$ \\
IIPs & $73.0 \pm 14.4$ & $14: 7$ & $6 / 21$ \\
CVD-ILDs & $74.9 \pm 6.7$ & $4: 4$ & $4 / 8$ \\
\hline
\end{tabular}

NSIP, and 1 with COP. CVD-ILDs included 4 patients with rheumatoid arthritis (RA), 3 with generalized scleroderma, and one with systemic lupus erythematosus (SLE).

The number of patients with a high anti-Cladosporium cladosporioides titer was significantly higher in ILDs compared to control subjects or BA (Table 1). Patients with a high antibody titer were observed in all of the three ILD groups (Table 2). Furthermore, five IPF, one NSIP, and no COP were included in IIPs with a high antibody titer, and three RA and one SLE in CVD-ILD exhibited a high antibody titer.

Age, sex, serum concentrations of LDH (lactate dehydrogenase) and sialylated carbohydrate antigen KL-6 were compared between ILD patients with high anti-Cladosporium cladosporioides antibody titer and other ILD patients. The ages of high antibody titer patients and others were $72.2 \pm 11.1$ and $71.3 \pm 14.8$, respectively. The male/female ratios of each group were $8 / 4$ and $12 / 10$, respectively. Serum concentrations of LDH in high antibody titer patients and others were $317.9 \pm 191.3 \mathrm{IU} / \mathrm{L}$ and $326.2 \pm 150.5 \mathrm{IU} /$ $\mathrm{L}$, respectively, and those of KL-6 were $1,755.7 \pm 1,856.0$ $\mathrm{U} / \mathrm{ml}$ and 1,736.1 $\pm 1,677.4 \mathrm{U} / \mathrm{ml}$, respectively. No significant difference was observed between each group.

Antibody titers to Cladosporium herbarum and Leptosphaerulina spp. were measured with the same sera as those used for Cladosporium cladosporioides in the ILD group. A significant correlation was observed between the antibody titers to Cladosporium cladosporioides and Cladosporium herbarum (Fig. 2A), but not between Cladosporium cladosporioides and Leptosphaerulina spp. (Fig. 2B).

\section{Discussion}

In this study, we showed a significantly higher tendency for a high anti-Cladosporium antibody titer in ILD
A

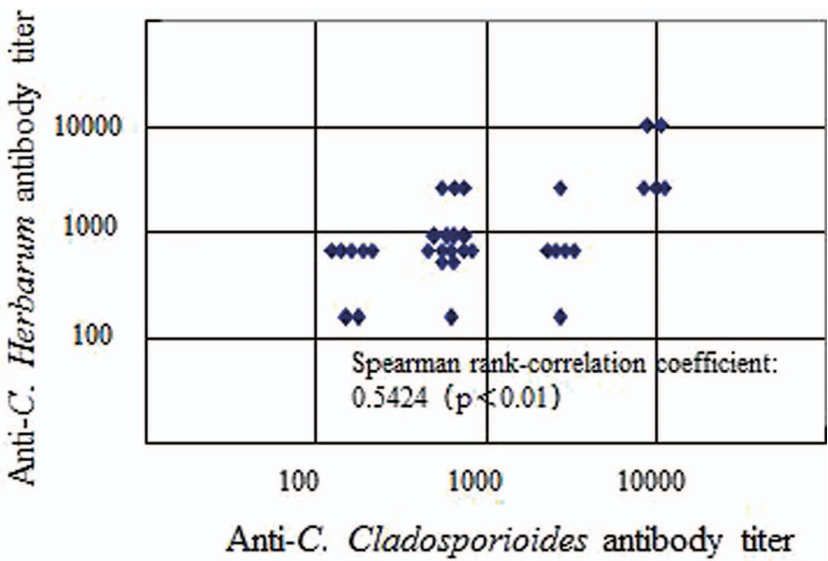

$\mathrm{B}$

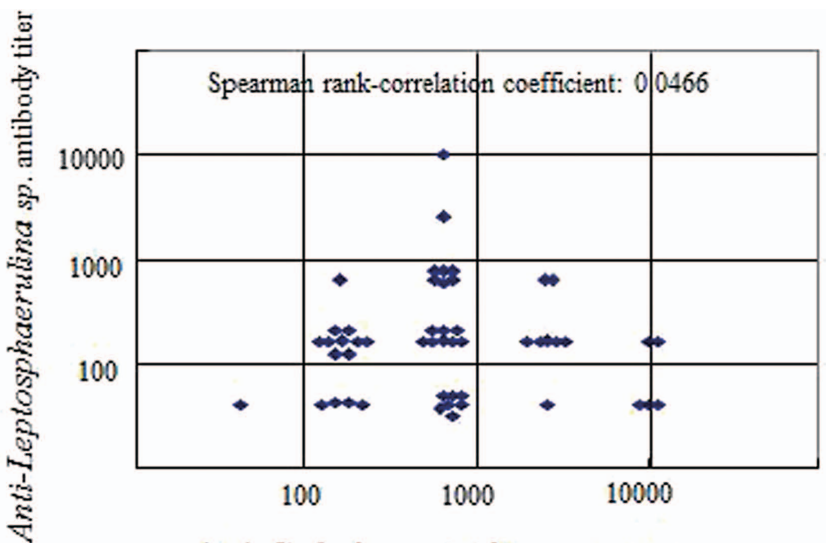

Anti-C.cladosporioides antibody titer

Fig. 2. Antibody titer correlation between Cladosporium cladosporioides and other fungi in the sera of ILD patients. Correlation of antibody titer to Cladosporium cladosporioides and Cladosporium herbarum or Leptosphaerulina spp. was investigated in ILD patients $(n=$ 34). A significant correlation was observed between the antibody titers to Cladosporium cladosporioides and Cladosporium herbarum (A), but not between Cladosporium cladosporioides and Leptosphaerulina spp. (B).

groups compared to patients with bronchial asthma or control subjects. This increase in antibody titer was observed not only in patients with HP, but also in those with IIPs and CVD-ILDs. These results indicate the potential of antiCladosporium antibody not only to separate possible Cladosporium-related HP patients from the large group of IIPs, but also to assist in the investigation of the role of fungal antigen in the pathogenesis of IPs. Furthermore, the absence of any difference for other biomarkers compared to that observed for antibody titer between high antibody titer patients and others indicates that a low antibody titer does not mean excluding the possibility of HP.

Achieving a correct diagnosis is difficult with ILDs. There are many ILDs with different etiologies and histological manifestations, and histological manifestation does not correspond to etiology according to a one-to-one relation 
(Schwarz 2003). For the correct diagnosis of IIPs, a comprehensive history of the patient is required to rule out nonidiopathic ILDs. Moreover, long-term observations of the patient are needed in addition to histological diagnosis based on surgically obtained lung specimens, and the low percentage of surgical biopsies in ILD patients has been debated (American Thoracic Society 2002). On the other hand, numerous sources of evidence suggest that environmental agents play a role in IIPs, although IIPs are categorized as ILDs without a known etiology. Interviews with patients and a search of medical records indicate a history of exposure to metal dusts, wood dusts, or some occupational settings suggestive of HP (Scott et al. 1990; Baumgartner 2000; Miyake 2005). In addition, HP cases indistinguishable from IIPs have recently been recognized (Chiba et al. 2009). Thus, a diagnosis system for the etiological factors is needed which involves lower invasiveness. An antibody assay, which has been used for the diagnosis of various diseases including CVDs, allergic and infectious diseases, could help obtain a correct diagnosis. For this purpose, it is important to find common antigens to indicate HP from the wide variety of HP antigens.

Cladosporium is a common fungus frequently encountered in most areas on the earth (Al-Doory 1984). The colony of the fungus appears gray to black or very dark green on dead plants, food, straw, soil, paint, textiles, and many other organic materials. Cladosporium, the spores of which are easily made airborne, has been known as an aero-allergen that induces BA (Cobe 1932), although it is not common to see this as positive in a RAST evaluation of patients with BA in Japan. Despite the prevalence of this fungus in our environment, this common antigen has received little attention in studies of the pathogenesis of HP compared with occupational or special environmental exposure, such as farmer's lung or bird fancier's lung, and discussion has focused mainly on the differentiation between the chronic form of classical HP and IIPs. We think it is important to consider that exposure to Cladosporium occurs ubiquitously in our living environment, and that even a careful examination of the history of a patient cannot distinguish HP from IIPs or remove the possibility of an HP factor in every IIP patient.

In this study, we measured the antibody titer to Cladosporium by IFA, which is a sensitive but not quantitative method to measure antibody titer compared to radioimmunoassay or enzyme immunoassay, although it is better than the Ouchterloney method in terms of sensitivity and quantity (Parratt et al. 1975). IFA measures the antibody response to all of the antigens in Cladosporium. Moreover, the use of a second antibody with wide selectivity to the human antibody subclass allows measurement of the total antibody titer to the Cladosporium antigen. Multiplicities in both antigen and antibody have been reported in HP, and there may be more than ten antigens in Cladosporium which are recognized as an allergen (Achatz 1995; Matsunaga et al. 2003). Thus, IFA is the best method at present to measure anti-Cladosporium antibody for the purpose of differentiating fungus-related IPs from IIPs. In the future, when the antigens and antibodies responsible for the diagnosis of HP are identified, a more sensitive and quantitative method should be developed.

Due to the huge and increasing number of environmental antigens, it is difficult to detect the immune responses to environmental antigens related to HP and to distinguish HP from IIPs. In this study, we showed possible antigenic cross-reactivity between Cladosporium cladosporioides and Cladosporium herbarum. Furthermore, common antigens between Cladosporium and Alternaria have been reported, which indicates that we measured antibody titer to common antigens of a wide variety of fungi, and fewer fungal antigens may therefore be needed to differentiate HP from IIPs, as in the case of the IgE titer in BA (Achatz 1995).

Several factors may relate to a high anti-Cladosporium antibody titer. First, a high concentration of fungal antigen or long-term exposure to fungal antigen may increase the antibody titer. High antibody titers were recorded for some co-workers working with an HP patient and for laboratory staff manipulating the fungus, and these data were not included in the control group in this study. Such cases indicate that high exposure to the fungus increases the antibody titer, and that this is not sufficient for the development of ILDs. Second, impairment of the fungal antigen clearance system may result in a high concentration of fungal antigen, as patients with bronchiectasis are sometimes infected with Aspergillus and other fungi. Although we did not measure the antibody titer in other disease groups such as COPD or bronchiectasis in this study, it is possible that these patients also have an increased antibody titer to fungal antigen. Third, sensitivity to antigens or an abnormality of the immune system could play roles in the high antibody titer to fungal antigen. An increase in antibody titers was observed in CVD-ILDs, although we expected that CVDILDs would be a negative control for idiopathic IPs.

In this study, we observed high anti-Cladosporium antibody titers in diverse types of ILDs. Although the mechanisms that increase the antibody level in humans may be different and specific for each disease, this increase in fungal antibody titer may indicate the role of fungi in the etiology of diseases. The roles of fungal antigens or fungal components in the development of ILDs have not been thoroughly studied. A study of ILDs has reported an increase in expression of toll-like receptor 9, the $\mathrm{CpG}$ receptor in nuclei, which reacts with the $\mathrm{CpG}$ motif in fungi (Meneghin 2008). Stimulation of toll-like receptor 9 induces proinflammatory cytokine release and the proliferation of lung fibroblasts (Meneghin 2008; Ramirez-Ortiz 2008). Additionally, it has been reported that fungi can induce the release of proinflammatory cytokine from alveolar macrophages, and inflammatory responses have been recorded in a murine model of arthritis and interstitial pneumonia through $\beta$-D-glucan and its receptor Dectin- 1 
(Yoshitomi 2005; Steele 2005). It is possible that fungi are involved in the pathogenesis of ILDs.

In conclusion, fungi commonly observed in our living environment such as Cladosporium could be involved in the development of ILDs. Therefore, the role of fungal stimulation in pulmonary inflammation and fibrosis needs further investigation in order to develop better treatment strategies for ILDs.

\section{Conflict of Interest}

We declare no conflict of interest.

\section{References}

Achatz, G., Oberkofler, H., Lechenauer, E., Simon, B., Unger, A., Kandler, D., Ebner, C., Prillinger, H., Kraft, D. \& Breitenbach, M. (1995) Molecular cloning of major and minor allergens of Alternaria alternata and Cladosporium herbarum. Mol. Immunol., 32, 213-227.

Al-Doory, Y. (1984) Airborne Fungi. In: Mould Allergy. Edited by Al-Doory, Y., Domson, J.F., Lea \& Febiger, Philadelphia, pp $27-40$.

American Thoracic Society. (2002) American Thoracic Society/ European Respiratory Society international multidisciplinary consensus classification of the idiopathic interstitial pneumonias. Am. J. Respir. Crit. Care Med., 165, 277-304.

Baumgartner, K.B., Samet, J.M., Coultas, D.B., Stidley, C.A., Hunt, W.C., Colby, T.V., Waldron, J.A. \& Collaborating Centers. (2000) Occupational and environmental risk factors for idiopathic pulmonary fibrosis: a multicenter case-control study. Am. J. Epidemiol., 152, 307-315.

Chiba, S., Okada, S., Suzuki, Y., Watanuki, Z., Mitsuishi, Y., Igusa, R. \& Sekii, T. (2009) Cladosporium species-related hypersensitivity pneumonitis in household environments. Inter. Med., 48, 363-367.

Cobe, H.M. (1932) Asthma due to a mold: hypersensitivity due to Cladosporium fulvum, Cooke. A case report. J. Allergy, 4, 389-391.

Fink, J.N., Ortega, H.G., Reynolds, H.Y., Cormier, Y.F., Fan, L.L., Franks, T.J., Kreiss, K., Kunkel, S., Lynch, D., Quirce, S., Rose, C., Schleimer, R.P., Schuyler, M.R., Selman, M., Trout, D. \& Yoshizawa, Y. (2005) Needs and opportunities for research in hypersensitivity pneumonitis. Am. J. Respir. Crit. Care Med., 171, 792-798.

Matsunaga, Y., Usui, Y. \& Yoshizawa, Y. (2003) TA-19, a novel protein antigen of Trichosporon asahii, in summer-type hypersensitivity pneumonitis. Am. J. Respir. Crit. Care Med., 167, 991-998.

Meneghin, A., Choi, E.S., Evanoff, H.L., Kunkel, S.L., Martinez, F.J., Flaherty, K.R., Toews, G.B. \& Hogaboam, C.M. (2008) TLR9 is expressed in idiopathic interstitial pneumonia and its activation promotes in vitro myofibroblast differentiation. Histochem. Cell Biol., 130, 979-992.

Miyake, Y., Sasaki, S., Yokoyama, T., Chida, K., Azuma, A., Suda, T., Kudoh, S., Sakamoto, N., Okamoto, K., Kobashi, G., Washio, M., Inaba, Y. \& Tanaka, H. (2005) Occupational and environmental factors and idiopathic pulmonary fibrosis in Japan. Ann. Occup. Hyg., 49, 259-265.

Parratt, D., Nielsen, K.H., Boyd, G. \& White, R.G. (1975) The quantitation of antibody in farmer's lung syndrome using a radioimmunoassay. Results of a clinical survey and comparison of three serological methods. Clin. Exp. Immunol., 20, 217-225.

Ramirez-Ortiz, Z.G., Specht, C.A., Wang, J.P., Chrono, K., Lee, C.K., Bartholomeu, D.C., Gazzinelli, R.T. \& Levitz, S.M. (2008) Toll-like receptor 9-dependent immune activation by unmethylated $\mathrm{CpG}$ motifs in Aspergillus fumigatus DNA. Infect. Immun., 76, 2123-2129.

Schwarz, M.I., King, T.E. \& Raghu, G. (2003) Approach to the evaluation and diagnosis of interstitial lung disease. In: Interstitial lung disease. $4^{\text {th }}$ ed. Edited by Schwarz, M.I., King, T.E., Decker, B.C., Hamilton, pp 1-30.

Scott, J., Johnston, I. \& Britton, J. (1990) What causes cryptogenic fibrosing alveolitis? A case-control study of environmental exposure to dust. Brit. Med. J., 301, 1015-1017.

Steele, C., Rapaka, R.R., Metz, A., Pop, S.M., Williams, D.L., Gordon, S., Kolls, J.K. \& Brown, G.D. (2005) The betaglucan receptor dectin-1 recognizes specific morphologies of Aspergillus fumigatus. PLoS Pathog., 1, e42. Epub 2005 Dec 9.

Yoshitomi, H., Sakaguchi, N., Kobayasi, K., Brown, G.D., Tagami, T., Sakihama, T., Hirota, K., Tanaka, S., Nomura, T., Miki, I., Gordon, S., Akira, S., Nakamura, T. \& Sakaguchi, S. (2005) A role for fungal $\beta$-glucans and their receptor Dectin- 1 in the induction of autoimmune arthritis in genetically susceptible mice. J. Exp. Med., 201, 949-960. 